\title{
COVID - 19 - A New Challenging Disease for Intensivists and Cardiologists. From Basic Science to the Clinical Setting.
}

\author{
Robert Skalik ${ }^{1,2}$
}

1. Wroclaw Medical University, Department of Physiology, Wroclaw, Poland

2. Specialist Hospital with Outpatients Clinics "Ventriculus", Leszno, Poland

\author{
Corresponding author: \\ Robert Skalik \\ Wroclaw Medical University, \\ Department of Physiology, \\ T. Chalubinskiego ST 10, \\ Postal Code 50-368,Wroclaw, Poland \\ email: robertskalik@tlen.pl
}

\section{Highlights}

A novel coronavirus (COVID-19) causing Acute Respiratory Distress Syndrome (SARS-CoV-2) was considered culpable for the huge number of lower respiratory tract infections leading to severe respiratory failure in many cases. However, the hospital reports show that the COVID -19 infestation may range from the asymptomatic or poorly symptomatic course with an almost bizarre deterioration of life parameters ("silent hypoxia") through to critically ill patients with multiple organ failure. Moreover, the coronavirus disease may cause lung damage in the asymptomatic COVID-19 positive patients who recover at home. The virus enters through angiotensin-converting enzyme 2 receptor (ACE2) widely expressed in the cardiovascular system and other organs, which can result in myocardial injury, myocarditis, acute myocardial infarction, heart failure, brain stroke, pneumonia and thromboembolic events. The COVID -19 - induced failure of central mechanisms controlling breathing and circulation may explain the mismatch between the clinical symptoms and the objective physiologic life parameters in many patients treated by intensivists or cardiologists. The severity of coronavirus disease is dependent on numerous factors including the magnitude of host immune response, the impairment of central and peripheral nervous system, age, comorbidities (e.g. diabetes, arterial hypertension, obesity, chronic heart failure) and presumably genetic proclivity. The brand-new treatment approach with use of alternative or experimental therapies such us Extracorporeal Membrane Oxygenation (ECMO), plasmapheresis, proinflammatory interleukins - targeted drugs, covalescent plasma transfusion, virus replication inhibitors is obviously needed in some COVID- 19 patients.

Keywords: COVID-19; symptoms; pathophysiologic mechanisms; cardiovascular diseases; new therapies

Citation: $\quad$ Skalik R. COVID - 19 - A New Challenging Disease for Intensivists and Cardiologists. From Basic Science to the Clinical Setting. International Cardiovascular Forum Journal 2020;20:2-4.DOI: 10.17987/icfj.v20i0.683

\section{Introduction}

At the end of 2019 in Chinese City Wuhan, a novel coronavirus (COVID-19) causing Severe Acute Respiratory Syndrome (SARS-CoV-2) was considered culpable of the enormous number of lower respiratory tract infections leading to severe respiratory complications. The virus has turned out to have a respectable potential to infect rapidly many people in China and subsequently trigger a global epidemic [1]. Since the beginning of March 2020, the COVID-19 pandemic has caused more than 13,000 deaths in Europe, almost $54 \%$ of which have occurred in Italy (in Poland, number of deaths has reached over 900 people since March 2020) [2]. The healthcare systems all over the world have been faced up with tremendous hospital admission rate and difficulty in the accurate and efficient diagnosing of such a high volume of patients suspected of the COVID-19 infection. The crushing majority of efforts to contain the COVID-19 pandemic has been focused on respiratory problems and securing enough ventilators. To make matters worse, doctors have promptly realized that the disease is insidious and has many faces. Much to the surprise of some intensivists, the effectiveness of conventional mechanical ventilation is below expectation or it does not work at all in many COVID-19 patients suffering from acute respiratory failure. According to the New York state and city officials, over $80 \%$ of COVID-19 patients placed on ventilators in New York City have died [3]. Many of them are dying while intubated on a ventilator. The increasing number of emergency physicians become aware that ventilators are not always helpful and Extracorporeal Membrane Oxygenation (ECMO) or other non-conventional therapies might be the last resort for some critically ill patients [4]. 
At present, we know that the COVID -19 infestation may range from the asymptomatic or poorly symptomatic course with an almost bizarre deterioration of life parameters (asymptomatic hypoxemia on pulse oximetry called "silent hypoxia") through to severely ill patients with multiple organ failure including lungs, kidneys, nervous system and other organs. Moreover, the coronavirus disease may also cause relevant lung damage in the asymptomatic COVID-19 positive patients as recently reported by Austrian doctors from University Clinic in Innsbruck. As more data come in from China, Italy and USA, it is believed that COVID-19 can also infect cardiovascular system, albeit in the majority of cases myocardial injury is more likely related to systemic consequences [5]. The virus binds and enters through angiotensin-converting enzyme 2 receptor (ACE2) widely expressed in heart, lungs, vessels and brain, which can result in systemic inflammation, multiorgan dysfunction and cardiovascular complications including myocardial injury, myocarditis, acute myocardial infarction, heart failure, dysrhythmias, brain stroke, acute pleuritis, pneumonia and venous thromboembolic events (Table 1) [6].

The pathophysiology of cardiovascular dysfunction in COVID-19 patients is probably a complex and multifactorial issue. The myocardial damage or cardiovascular failure due to the coronavirus disease can be related to the direct viral intrusion into the cardiac muscle, the excessive immediate host immune response to the virus ("cytokine storm"), arterial blood hypoxemia and acidosis in the wake of the alveolar-capillary membrane damage, the impairment of brain cardiorespiratory controls and lung-heart interaction during the acute inflammatory process that stems primarily from the lungs. It was previously confirmed on the animal model that experimentally-induced pleurisy may cause myocardial damage probably via activation of immunological system as soon as 24 hours since the onset of acute inflammatory response [7]

There are several physiologic and pathophysiologic premises demonstrating that some of the weird prodromal symptoms (smell and taste sensations) or lack of symptoms (lack of dyspnoea in patients with relevant hypoxemia) in some COVID-19 patients might be caused by damage to the central (CNS) and peripheral nervous system [8]. The lung ventilation is controlled by the brain (central chemoreceptors in the medulla oblongata, brain cortex and hypothalamus), peripheral chemoreceptors (in the aorta and carotid vessels) and signalling from the lung stretch receptors to the brain stem via afferent vagal nerve endings. COVID-19 causes smell and taste disorders that probably originate from some focal injury in CNS and/or olfactory and taste receptors. It is likely that the virus may also impair "breathlessness sensations" centre in the brain cortex (insula), central and peripheral chemoreceptors as it does taste and smell receptors $[8,9]$. Subsequently, the coronavirus may cause missignalling between the chemoreceptors, autonomic controls of breathing and circulation and other cerebral structures. For this reason, the severely ill COVID-19 patients may not feel dyspnoea, even though their objective life parameters dramatically deteriorate and might be indicative of acute cardiopulmonary failure. Many brain areas can be affected by the virus including cerebrum, vagal nerve, paraventricular nucleus of hypothalamus, brain stem and others. Some coronaviruses have been reported to spread via

\section{Table 1. COVID-19-induced cardiovascular complications}

Comorbidity

Myocardial Injury and Myocarditis

Acute Myocardial Infarction

Heart Failure and Cardiomyopathy

Arrhythmias

Shock and Cardiac Arrest

Venous Thromboembolic Events

a synapse-connected route to the medullary cardiorespiratory center from the mechanoreceptors and chemoreceptors in the lung and lower respiratory airways $[8,9]$.

The viral infection may have strong affinity to the chemosensitive area in the brain stem (chemoreceptors) involved in chemoreflex - induced hyperventilation response to hypercapnia [10]. It was corroborated by experimental models that intranasally injected lethal avian influenza $A(H 5 N 1)$ virus can replicate in pontomedullary chemosensitive neurons of the brain and this way depress hypercapnic ventilatory response [10]. According to the previous studies, the enhanced chemoreflex as observed in patients with chronic heart failure (CHF) may lead to hyperpnea and exercise intolerance [11]. It can be hypothesized that the coronavirus may impair brain stem reflex regulating minute ventilation and the response of chemoreceptors to the changing arterial blood gas levels (no shortness of breath despite relevant hypoxemia). However, this issue needs further investigations on the experimental animal models or in patients who have just recovered from COVID -19 (the post-treatment assessment of chemoreflex sensitivity in the laboratory conditions).

The sudden deterioration of clinical status in several COVID-19 patients during intubation as reported by some intensivists may also indirectly indicate the impairment of chemoreflex that is mediated by vagal nerve endings. The autonomic nervous system (ANS) that controls respiration and heart function is a system of "interrelated vessels". ANS has ability to converge and diverge information flowing in from various parts of human body (lungs, upper respiratory tract, cardiac muscle, vessels, hormonal system, immune system, urinary system, skin, muscles, gastrointestinal tract). At present, we know that many of the above - mentioned entities can be attacked by the virus. The weird reflex response to tracheal tube insertion while connecting the COVID-19 patients with ventilators may lead to worsening of the respiratory function through abnormal signalling between vagal nervous endings in trachea and brain stem due to the coronavirus intrusion into CNS and peripheral nerve endings. Besides, the coronavirus and virus-related host immune response can devastate the alveolar-capillary membrane that is necessary for gas exchange in the lungs. Hence, the conventional mechanical ventilation in some most severely ill COVID-19 patients can be even counterproductive and add to lung tissue damage [12]. It has been reported that the use of oxygen masks with the unusual body position ("prone position") and ECMO are more helpful than the mechanical ventilation in many COVID-19 patients with respiratory failure.

The clinical observations and better understanding of the 
complex physiologic and pathophysiologic mechanisms implicated in the deleterious COVID-19 effect on the various human body systems should encourage doctors and decisionmakers to revise the treatment guidelines for some ICU patients and use the alternative therapies such as ECMO, plasmapheresis, anti-interleukin monoclonal antibodies, viral replication inhibitors or convalescent plasma in the critically ill patients [13]

\section{Declarations of Interest}

The author declares no conflicts of interest.

\section{Acknowledgements}

The author states that he abides by the "Requirements for Ethical Publishing in Biomedical Journals". [14]

\section{References}

1. Krajewska J, Krajewski W, Zub Ket al. COVID-19 in otolaryngologist practice: a review of current knowledge. Eur Arch Otorhinolaryngol. 2020. DOI: 10.1007/s00405-020-05968-y

2. Radovanovic D, Rizzi M, Pini S et al. Helmet CPAP to Treat Acute Hypoxemic Respiratory Failure in Patients with COVID-19: A Management Strategy Proposal. J Clin Med. 2020;9: 1191. DOI: 10.3390/jcm9041191

3. Richardson S, Hirsch JS, Narasimhan M et al. Presenting Characteristics, Comorbidities, and Outcomes Among 5700 Patients Hospitalized With COVID-19 in the New York City Area. JAMA 2020. DOI: 10.1001/ jama.2020.6775

4. Gattinoni L, Coppola S, Cressoni M et al. Covid-19 Does Not Lead to a "Typical" Acute Respiratory Distress Syndrome. Am J Respir Crit Care Med. 2020. DOI:10.1164/rccm.202003-0817LE

5. Deng $Q, H u$ B, Zhang $Y$ et al. Suspected myocardial injury in patients with COVID-19: Evidence from front-line clinical observation in Wuhan, China. Int J Cardiol. 2020. DOI: 10.1016/j.ijcard.2020.03.087

6. Long B, Brady WJ, Koyfman A et al. Cardiovascular complications in COVID-19. Am J Emerg Med. 2020. DOI: 10.1016/j.ajem.2020.04.048

7. Całkosiński I, Skalik R, Borodulin-Nadzieja L et al. Influence of Inflammatory Reaction on Blood Concentration of Cholesterol And Other Biochemical Values With Regard To Cardiac Muscle Damage in Rats. Bull Vet Inst Pulawy. 2004; 48: 477-480.

8. Netland J, Meyerholz DK,Moore S et al. Severe acute respiratory syndrome coronavirus infection causes neuronal death in the absence of encephalitis in mice transgenic for human ACE2. J Virol. 2008;82:7264-7275. DOI: 10.1128/JVI.00737-08

9. Li YC, Bai WZ, Hashikawa T. The neuroinvasive potential of SARS-CoV2 may play a role in the respiratory failure of COVID-19 patients. J Med Virol. 2020. DOI: 10.1002/jmv.25728

10. Zhuang J, Zang N, Ye C et al. Lethal avian influenza A (H5N1) virus replicates in pontomedullary chemosensitive neurons and depresses hypercapnic ventilatory response in mice. Am J Physiol Lung Cell Mol Physiol. 2019;316:L525-L536. DOI:10.1152/ajplung.00324.2018

11. Ponikowski $P$, Chua TP, Piepoli $M$ et al. Augmented periphera chemosensitivity as a potential input to baroreflex impairment and autonomic imbalance in chronic heart failure. Circulation. 1997;96:25862594. DOI:10.1161/01.cir.96.8.2586.

12. Beitler JR, Malhotra A and Thomson BT. Ventilator-Induced Lung Injury. Clin Chest Med. 2016; 37: 633-646. DOI: 10.1016/j.ccm.2016.07.004

13. Rizzo P, Vieceli Dalla Sega F, Fortini F et al. COVID-19 in the heart and the lungs: could we „Notch” the inflammatory storm? Basic Res Cardiol. 2020 ;115:31. DOI: 10.1007/s00395-020-0791-5

14. Shewan LG, Coats AJS, Henein MY. Authors' Responsibilities and Ethica Publishing. International Cardiovascular Forum Journal 2018;13:3-4 DOI:10.17987/icfj.vl3i0.525 